TRANSACTIONS OF THE

AMERICAN MATHEMATICAL SOCIETY

Volume 363, Number 10, October 2011, Pages 5367-5379

S 0002-9947(2011)05299-2

Article electronically published on May 12, 2011

\title{
LORENTZIAN MANIFOLDS ISOMETRICALLY EMBEDDABLE IN $\mathbb{L}^{N}$
}

\author{
O. MÜLLER AND M. SÁNCHEZ
}

\begin{abstract}
In this article, the Lorentzian manifolds isometrically embeddable in $\mathbb{L}^{N}$ (for some large $N$, in the spirit of Nash's theorem) are characterized as a subclass of the set of all stably causal spacetimes; concretely, those which admit a smooth time function $\tau$ with $|\nabla \tau|>1$. Then, we prove that any globally hyperbolic spacetime $(M, g)$ admits such a function, and, even more, a global orthogonal decomposition $M=\mathbb{R} \times S, g=-\beta d t^{2}+g_{t}$ with bounded function $\beta$ and Cauchy slices.

In particular, a proof of a result stated by C.J.S. Clarke is obtained: any globally hyperbolic spacetime can be isometrically embedded in Minkowski spacetime $\mathbb{L}^{N}$. The role of the so-called "folk problems on smoothability" in Clarke's approach is also discussed.
\end{abstract}

\section{INTRODUCTION}

A celebrated theorem by J. Nash [14] states that any $C^{3}$ Riemannian manifold can be isometrically embedded in any open subset of some Euclidean space $\mathbb{R}^{N}$ for large $N$. Greene [9] and Clarke [7] showed independently, by means of simple algebraic reasonings, that Nash's theorem can be extended to indefinite (even degenerate) metrics, that is, any semi-Riemannian manifold can be smoothly isometrically embedded in any open subset of semi-Euclidean space $\mathbb{R}_{s}^{N}$ for large enough dimension $N$ and index $s$. Moreover, they also reduced the Nash value for $N$ : Greene by using the implicit function theorem by Schwartz [18, and Clarke by means of a technique inspired by Kuiper [11, which yields $C^{k}$ isometric embeddings with $3 \leq k<\infty$.

Nevertheless, a new problem appears when a semi-Riemannian manifold of index $s$ is going to be embedded in a semi-Euclidean space of the same index $\mathbb{R}_{s}^{N}$. We will focus on the simplest case $s=1$, i.e., the isometric embedding of a Lorentzian manifold $(M, g)$ in Minkowski spacetime $\mathbb{L}^{N}$. Such an embedding will not exist in general; for example, the existence of a causal closed curve in $M$ contradicts the possibility of an embedding in $\mathbb{L}^{N}$. So, the first task is to characterize the class of isometrically embeddable spacetimes. This is the role of our first result (Section 3):

Theorem 1.1. Let $(M, g)$ be a Lorentzian manifold. The following assertions are equivalent:

(i) $(M, g)$ admits an isometric embedding in $\mathbb{L}^{N}$ for some $N \in \mathbb{N}$.

Received by the editors July 19, 2009 and, in revised form, December 18, 2009.

2010 Mathematics Subject Classification. Primary 53C50, 53C12, 83E15, 83C20.

Key words and phrases. Causality theory, globally hyperbolic, isometric embedding, conformal embedding. 
(ii) $(M, g)$ is a stably causal spacetime with a steep temporal function, i.e., a smooth function $\tau$ such that $g(\nabla \tau, \nabla \tau) \leq-1$.

Again, this theorem is carried out by using some simple arguments, which essentially reduce the hardest problem to the Riemannian case. So, this result (and the subsequent ones on isometric embeddings) is obtained under the natural technical conditions which come from the Riemannian setting: (a) $(M, g)$ must be $C^{k}$ with $3 \leq k \leq \infty$, and all the other elements will be as regular as permitted by $k$, and (b) the smallest value of $N$ is $N=N_{0}(n)+1$, where $n$ is the dimension of $M$ and $N_{0}(n)$ is the optimal bound in the Riemannian case (see [10] for a recent summary on this bound). We will not care about the local problem (see [9], a summary in Lorentzian signature can be found in [19]); recall also that, locally, any spacetime fulfills condition (ii). So, the main problem we will consider below is the existence of a global steep temporal function as stated in (ii).

It is known that any stably causal spacetime admits a time function, which can be smoothed into a temporal one $\tau$ (see Section 2 for definitions and background). Nevertheless, the condition of being steep, $|\nabla \tau| \geq 1$ cannot be fulfilled for all stably causal spacetimes. In fact, a simple counterexample, which works even in the causally simple case, is provided below (Example [3.3). Notice that causal simplicity is the level in the standard causal hierarchy of spacetimes immediately below global hyperbolicity. So, the natural question is to wonder if any globally hyperbolic spacetime admits a steep temporal function $\tau$.

The existence of embeddings in $\mathbb{L}^{N}$ for globally hyperbolic spacetimes was stated by Clarke [7, Sect. 2]. In his approach, a function $f: M \rightarrow \mathbb{L}^{2}$ with a similar role to the steep temporal function above is used. Nevertheless, as in other papers of that epoch, his construction of $f$ is affected by the so-called "folk problems" of smoothability of causally-constructed functions. So, as will be discussed in the Appendix, if Clarke's proof is completed, then a new type of causally-constructed functions will be shown to be smooth (or at least smoothable).

Apart from the consequence of the embedding in $\mathbb{L}^{N}$, the existence of a steep temporal $\tau$ is relevant for the structure of globally hyperbolic spacetimes. In fact, both questions, problems of smoothability and structure of globally hyperbolic spacetimes, are linked since Geroch's landmark about topological splittings [8]. More precisely, recently any globally hyperbolic spacetime $(M, g)$ has been proved to admit a Cauchy orthogonal decomposition

$$
M=\mathbb{R} \times S, \quad g=-\beta d \mathcal{T}^{2}+g_{\mathcal{T}},
$$

where $\beta>0$ is a function on $M, g_{\mathcal{T}}$ is identifiable to a Riemannian metric on $S_{\mathcal{T}}:=\{\mathcal{T}\} \times S$ smoothly varying with $\mathcal{T}$, and each slice $S_{\mathcal{T}}$ becomes a Cauchy hypersurface [4. This result, which improves Geroch's topological splitting $M \cong$ $\mathbb{R} \times S$ as both a differentiable and orthogonal one, is proved by showing that, starting at Geroch's Cauchy time function, one could obtain a Cauchy smooth time function with timelike gradient. Now, recall that, if this Cauchy temporal function is steep, then the function $\beta$ is upper bounded by one (Lemma 3.5). One of us suggested possible analytical advantages of a strengthened decomposition (1.1), where additional conditions on the elements $\beta, g_{\mathcal{T}}$ are imposed [13. In particular, 
such a decomposition is called a b-decomposition there if the function $\beta$ (the lapse in relativist's terminology) is bounded. Our next result is then (Section 4):

Theorem 1.2. Any globally hyperbolic spacetime admits a steep Cauchy temporal function $\mathcal{T}$ and, so, a Cauchy orthogonal decomposition (1.1) with (upper) bounded function $\beta$.

Remark 1.3. From the technical viewpoint, the decomposition (1.1) was carried out in 4 by proving the existence of a Cauchy temporal function; moreover, a simplified argument shows the existence of a temporal function in any stably causal spacetime (4, see also the discussion in [17]). Our proof is completely self-contained, as it re-proves the existence of the Cauchy temporal function with different and somewhat simpler arguments, as well as a stronger conclusion. Nevertheless, we use some technical elements (remarkably, Proposition 4.2) which hold in the globally hyperbolic case, but not in the stably causal ond 1

Summing up, we emphasize the following consequences of previous theorems (for the second one also recall Proposition 2.1).

Corollary 1.4. (1) Any globally hyperbolic spacetime can be isometrically embedded in some $\mathbb{L}^{N}$.

(2) A Lorentzian manifold is a stably causal spacetime if and only if it admits a conformal embedding in some $\mathbb{L}^{N}$. In this case, there is a representative of its conformal class whose time-separation (Lorentzian distance) function is finite-valued.

Notice also that, as an immediate consequence, a stably causal spacetime is not globally hyperbolic if and only if it is conformal to a spacetime non-isometrically embeddable in $\mathbb{L}^{N}$ (see Example 3.3).

After some preliminaries in the next section, Sections 3 and 4 are devoted, respectively, to prove Theorems 1.1 and 1.2 as well as to discuss its optimality and consequences. From the technical viewpoint, it is worth pointing out the introduction of two elements in the first part of Section 4 a semi-local temporal function for subsets of type $J^{ \pm}(p) \cap J^{\mp}(S)$ (Proposition 4.2) and fat cone coverings for any Cauchy hypersurface $S$ (Proposition 4.4). Finally, in the Appendix, Clarke's technique for globally hyperbolic spacetimes is discussed, and new causal problems on smoothability, which may have their own interest, are suggested.

\section{Preliminaries}

In what follows, any semi-Riemannian manifold will be $C^{k}$, with $3 \leq k \leq \infty$ as in Nash's theorem, and will be assumed to be connected without loss of generality. Any geometric element on the manifold will be called smooth if it has the highest order of differentiability allowed by $k$. For an immersion $i: M \rightarrow \bar{M}$ only injectivity of each $d i_{p}, p \in M$ is required; the injectivity of $i$, as well as being a homeomorphism onto its image, are required additionally for $i$ to be an embedding.

Our notation and conventions on causality will be standard as, for example, in [2] or [15]. Nevertheless, some terminology on the solution of the so-called "folk problems of smoothability" introduced in [3, 4] are also used here (see [12] for a review). In particular, a Lorentzian manifold $(M, g)$ is a manifold $M$ endowed with a metric tensor $g$ of index one $(-,+, \ldots,+)$, a tangent vector $v \in T_{p} M$ in $p \in M$,

\footnotetext{
${ }^{1}$ Notice also that only $C^{1}$ differentiability is needed for these results.
} 
is timelike (resp. spacelike; lightlike; causal) when $g(v, v)<0$ (resp. $g(v, v)>0$; $g(v, v)=0$ but $v \neq 0 ; v$ is timelike or lightlike); so, following [12, the vector 0 will be regarded as non-spacelike and non-causal - even though this is not by any means the unique convention in the literature. For any vector $v$, we write $|v|:=\sqrt{|g(v, v)|}$. A spacetime is a time-orientable Lorentzian manifold, which will be assumed to be time-oriented (choosing any of its two time-orientations) when necessary; of course, the choice of the time-orientation for submanifolds conformally immersed in $\mathbb{L}^{N}$ will agree with the induced from the canonical time-orientation of $\mathbb{L}^{N}$. The associated time-separation or Lorentzian distance function will be denoted by $d, d(p, q):=\sup _{c \in \Omega(p, q)} l(c)$ where the supremum is taken over the space $\Omega(p, q)$ of future-directed causal $C^{1}$ curves from $p$ to $q$ parametrized over the unit interval (if this space is empty, $d$ is defined equal to 0 ), and $l(c):=\int_{0}^{1}|\dot{c}(t)| d t$ for such a curve. The following elements of causality must be taken into account (they are explained in detail in [12]).

- A time function $t$ on a spacetime is a continuous function which increases strictly on any future-directed causal curve. Recently [4, it has been proved that this is also equivalent to the existence of a temporal function $\tau$, i.e., a smooth time function with everywhere past-directed timelike gradient $\nabla \tau$. This also ensures the folk claim that, for a spacetime, the existence of a time funtion is equivalent to be stably causal (i.e., if the lightcones of the spacetime are slightly opened, then it remains causal), see [17, Fig. 2, Th. 4.15, Rem. 4.16] or [12, Th. 3.56]. In the present paper, a temporal function will be called steep if $|\nabla \tau| \geq 1$; as we will see, not all stably causal spacetimes admit a steep temporal function.

- After stable causality, the two next steps in the so-called causal ladder or causal hierarchy of spacetimes are: causal continuity (the volume functions $t^{ \pm}(p)=\mu\left(I^{ \pm}(p)\right), p \in M$ are time functions for one, and then for all, measure associated to any auxiliary semi-Riemannian metric such that $\mu(M)<\infty$ ) and causal simplicity (the spacetime is causal with closed $J^{+}(p), J^{-}(q)$ for all $\left.p\right)$. A spacetime is called globally hyperbolic if it is causal and the intersections $J^{+}(p) \cap J^{-}(q)$ are compact for all $p, q \in M$ (for the last two definitions, notice [6]). Globally hyperbolic spacetimes are the most relevant from both the geometric and physical viewpoints, and lie at the top of the causal hierarchy .

- A time or temporal function is called Cauchy if it is onto on $\mathbb{R}$ and all its level hypersurfaces are Cauchy hypersurfaces (i.e., topological hypersurfaces crossed exactly once by any inextensible timelike curve). A classical theorem by Geroch [8] asserts the equivalence between: (i) to be globally hyperbolic, (ii) to admit a Cauchy hypersurface, and (iii) to admit a Cauchy time function. Moreover, the results in 3, 4 also ensure the equivalence with: (iv) to admit a (smooth) spacelike Cauchy hypersurface, and (v) to admit a Cauchy temporal function $\mathcal{T}$. As a consequence, the full spacetime admits an orthogonal Cauchy decomposition as in (1.1).

- Further properties have been achieved [5]: any compact acausal spacelike submanifold with boundary can be extended to a (smooth) spacelike Cauchy hypersurface $\Sigma$, and any such $\Sigma$ can be regarded as a slice $\mathcal{T}=$ constant for a suitable Cauchy orthogonal decomposition (1.1). Apart 
from the obvious interest in the foundations of classical General Relativity, such results have applications in fields such as the wave equation or quantization, see for example [1, 16].

The following simple results are useful for the discussions below.

Proposition 2.1. Let $(M, g)$ be a spacetime.

(1) If $\tau$ is a temporal function, then there exists a conformal metric $g^{*}=\Omega g$, $\Omega>0$, such that $\tau$ is steep.

(2) If $\mathcal{T}$ is a Cauchy temporal function and $\tau$ is a temporal function, then $\mathcal{T}+\tau$ is a Cauchy temporal function. Moreover, $\mathcal{T}+\tau$ is steep if either $\tau$ or $\mathcal{T}$ is.

Proof. (1) As $\nabla^{*} \tau=\nabla \tau / \Omega$, choose any $\Omega \leq|\nabla \tau|^{2}$.

(2) $\mathcal{T}+\tau$ is temporal (and steep, if so is any of the two functions) because of the reversed triangle inequality. In order to check that its level hypersurfaces are Cauchy, consider any future-directed timelike curve $\gamma:\left(a_{-}, a_{+}\right) \rightarrow M$. It is enough to check that $\lim _{s \rightarrow \pm a}(\mathcal{T}+\tau)(\gamma(s))= \pm \infty$. But this is obvious, because $\lim _{s \rightarrow \pm a} \mathcal{T}(\gamma(s))= \pm \infty$ (as $\mathcal{T}$ is Cauchy) and $\tau(\gamma(s))$ is increasing.

\section{Characterization of isometrically Embeddable LORENTZIAN MANIFOLDS}

Proposition 3.1. Let $(M, g)$ be a Lorentzian manifold. If there exists a conformal immersion $i: M \rightarrow \mathbb{L}^{N}$, then $(M, g)$ is a stably causal spacetime.

Moreover, if $i$ is an isometric immersion, then: (a) the natural time coordinate $t=x^{0}$ of $\mathbb{L}^{N}$ induces a steep temporal function on $M$, and (b) the time-separation $d$ of $(M, g)$ is finite-valued.

Proof. Notice that $x^{0} \circ i$ is trivially smooth and also a time function (as $x^{0}$ increases on $i \circ \gamma$, where $\gamma$ is any future-directed causal curve in $M$ ), which proves stable causality.

If $i$ is isometric, then $\left|\nabla\left(x^{0} \circ i\right)\right| \geq 1$ because, at each $p \in M, \nabla\left(x^{0} \circ i\right)_{p}$ is the projection of $\nabla x_{i(p)}^{0}$ onto the tangent space $d i\left(T_{p} M\right)$, and its orthogonal $\operatorname{di}\left(T_{p} M\right)^{\perp}$ in $T_{i(p)} \mathbb{L}^{N}$ is spacelike. This proves (a); for (b) notice that the finiteness of $d$ is an immediate consequence of the finiteness of the time-separation $d_{0}$ on $\mathbb{L}^{N}$ and the straightforward inequality $d(p, q) \leq d_{0}(i(p), i(q))$ for all $p, q \in M$.

Remark 3.2. As a remarkable difference with the Riemannian case, Proposition 3.1 yields obstructions for the existence of both conformal and isometric immersions in $\mathbb{L}^{N}$. In particular, non-stably causal spacetimes cannot be conformally immersed, and further conditions on the time-separation are required for the existence of an isometric immersion. In fact, it is easy to find even causally simple spacetimes splitted as in (1.1) (with levels of $\mathcal{T}$ non-Cauchy) which cannot be isometrically immersed in $\mathbb{L}^{N}$, as the following example shows.

Example 3.3. Let $M=\left\{(x, t) \in \mathbb{R}^{2}: x>0\right\}, g=\left(d x^{2}-d t^{2}\right) / x^{2}$. This is conformal to $\mathbb{R}^{+} \times \mathbb{R} \subset \mathbb{L}^{2}$ and, thus, causally simple. It is easy to check that $d(p, q)=\infty$ for $p=(1,-2), q=(1,2)$ (any sequence of causal curves $\left\{\gamma_{m}\right\}_{m}$ connecting $p$ and $q$ whose images contain $\{(1 / m, t):|t|<1\}$ will have diverging lengths). Thus, $(M, g)$ cannot be isometrically immersed in $\mathbb{L}^{N}$. 
Recall that this example can be generalized, taking into account that a stably causal spacetime is non-globally hyperbolic if and only if it is conformal to a spacetime with an infinite-valued time-separation (this holds for all strongly causal spacetimes, see [2, Th. 4.30]). So, in the conformal class of any non-globally hyperbolic spacetime, there are spacetimes non-isometrically inmersable in $\mathbb{L}^{N}$.

Nash's theorem will be essential for the proof of the following result.

Proposition 3.4. If a spacetime $(M, g)$ admits a steep temporal function $\tau$, then it can be isometrically embedded in $\mathbb{L}^{N}$ for some $N$.

For the proof, recall first.

Lemma 3.5. If a spacetime $(M, g)$ admits a temporal function $\tau$, then the metric $g$ admits an orthogonal decomposition

$$
g=-\beta d \tau^{2}+\bar{g},
$$

where $\beta=|\nabla \tau|^{-2}$ and $\bar{g}$ is a positive semi-definite metric on $M$ with radical spanned by $\nabla \tau$.

In particular, if $\tau$ is steep, then $\beta \leq 1$.

Proof. The orthogonal decomposition (3.1) follows by taking $\bar{g}$ as the trivial extension of $\left.g\right|_{(\nabla \tau)^{\perp}}$ to all $T M$. To determine the value of $\beta$, recall that $d \tau(\nabla \tau)=$ $g(\nabla \tau, \nabla \tau)=-\beta(d \tau(\nabla \tau))^{2}$.

Proof of Proposition 3.4. Consider the orthogonal decomposition in Lemma 3.5. Even though $M$ does not need to split as a product $\mathbb{R} \times S$ (in an open subset of $\mathbb{L}^{n}$, the vector field $\nabla \tau$ may be incomplete and the topology of the level sets may change), we can rewrite (3.1) as

$$
g=-\beta d \tau^{2}+g_{\tau}
$$

where each $g_{\tau_{0}}$ is Riemannian metric on the slice $S_{\tau_{0}}=\tau^{-1}\left(\tau_{0}\right)$ varying smoothly with $\tau_{0}$. Moreover, each $p \in M$ will be written as $(\tau, x)$ where $x \in S_{\tau(p)}$.

Now, consider the auxiliary Riemannian metric

$$
g_{R}:=(4-\beta) d \tau^{2}+g_{\tau} .
$$

By Nash's theorem, there exists an isometric embedding $i_{\text {nash }}:\left(M, g_{R}\right) \hookrightarrow \mathbb{R}^{N_{0}}$. Then, a simple computation shows that the required isometric embedding $i$ : $(M, g) \hookrightarrow \mathbb{L}^{N_{0}+1}$ is just:

$$
i(\tau, x)=\left(2 \tau, i_{\text {nash }}(\tau, x)\right) .
$$

Remark 3.6. (1) From the proof, it is clear that the hypotheses on steepness can be weakened just by assuming that $\nabla \tau$ is lower bounded by some positive function $\epsilon(\tau)>0$. In fact, this is equivalent to requiring $\beta(\tau, x) \leq A(\tau)^{2}:=1 / \epsilon(\tau)$, and the proof would work by taking $g_{R}:=\left(4 A(\tau)^{2}-\beta\right) d \tau^{2}+g_{\tau}$ and $i(\tau, x)=$ $\left(2 \int_{0}^{\tau} A(s) d s, i_{\text {nash }}(\tau, x)\right)$. Nevertheless, no more generality would be obtained in this case because of the following two different arguments: (a) it is easy to check that, if this weaker condition holds, then a suitable composition $\hat{\tau}=f \circ \tau$ for some increasing function $f$ on $\mathbb{R}$ would be steep and temporal, and (b) the existence of a steep temporal function would be ensured by taking the isometric embedding $i: M \hookrightarrow \mathbb{L}^{N}$ and restricting the natural coordinate $t=x^{0}$ as in Proposition 3.1.

(2) Notice that Proposition 3.1 yields a necessary condition for the existence of an isometric embedding and Proposition 3.4 a sufficient one. Both together prove 
trivially Theorem 1.1, as well as Corollary 1.4(2) (notice also Proposition 2.1(1)). Recall that, as a difference from Nash's theorem, Proposition 3.4 does not allow us to prove that the spacetime is isometrically embedded in an arbitrarily small open subset, which cannot be expected now (notice that $d(p, q) \leq d_{0}(i(p), i(q)) \leq$ $\left.\left|x^{0}(i(p))-x^{0}(i(q))\right|\right)$.

\section{The Cauchy orthogonal B-Decomposition of any gLobally HYPERBOLIC SPACETIME}

In order to obtain a steep Cauchy temporal function in a globally hyperbolic spacetime, Proposition 2.1(2) reduces the problem to find a steep temporal function (not necessarily Cauchy), as the existence of a Cauchy temporal function is ensured in [4. Nevertheless, we will prove directly the existence of a steep Cauchy temporal function $\mathcal{T}$, proving Theorem 1.2 independently of the results in 4] (recall Remark 1.3).

So, in what follows $(M, g)$ will be a globally hyperbolic spacetime, and we will assume that $t$ is a Cauchy time function as given by Geroch [8]. The following notation will also be used here. Regarding $t$,

$$
T_{a}^{b}=t^{-1}([a, b]), \quad S_{a}=t^{-1}(a) .
$$

For any $p \in M, j_{p}$ is the function

$$
q \mapsto j_{p}(q)=\exp \left(-1 / d(p, q)^{2}\right) .
$$

For any $A, B \subset M$,

$$
J(A, B):=J^{+}(A) \cap J^{-}(B),
$$

in particular, $J(p, S):=J^{+}(p) \cap J^{-}(S)$ for $S$ any (Cauchy) hypersurface.

4.1. Some technical elements. In the next two propositions we will introduce a pair of technical tools for the proof. But, first, consider the following straightforward lemma, which will be invoked several times.

Lemma 4.1. Let $\tau$ be a function such that $g(\nabla \tau, \nabla \tau)<0$ in some open subset $U$ and let $K \subset U$ compact. For any function $f$ there exists a constant $c$ such that $g(\nabla(f+c \tau), \nabla(f+c \tau))<-1$ on $K$.

Proof. Notice that at each $x$ in the compact subset $K$ the quadratic polynomial $g(\nabla(f(x)+c \tau(x)), \nabla(f(x)+c \tau(x)))$ becomes smaller than -1 for some large $c$.

The following "cone semi-time function" will be useful from a technical viewpoint.

Proposition 4.2. Let $S$ be a Cauchy hypersurface, $p \in J^{-}(S)$. For all neighborhood $V$ of $J(p, S)$ there exists a smooth function $\tau \geq 0$ such that:

(i) $\operatorname{Supp} \tau \subset V$.

(ii) $\tau>1$ on $S \cap J^{+}(p)$.

(iii) $\nabla \tau$ is timelike and past-directed in $\operatorname{Int}\left(\operatorname{Supp}(\tau) \cap J^{-}(S)\right)$.

(iv) $g(\nabla \tau, \nabla \tau)<-1$ on $J(p, S)$. 
Proof. Let $t$ be a Cauchy time function such that $S=S_{a}:=t^{-1}(a)$, and let $K \subset V$ be a compact subset such that $J\left(p, S_{a}\right) \subset \operatorname{Int}(K)$. Compactness guarantees the existence of some $\delta>0$ such that: for every $x \in K$ there exists a convex neighborhood $U_{x} \subset V$ with $\partial^{+} U_{x} \subset J^{+}\left(S_{t(x)+2 \delta}\right)$, where $\partial^{+} U_{x}:=\partial U_{x} \cap J^{+}(x)$. Now, choose $a_{0}<a_{1}:=t(p)<\cdots<a_{n}=a$ with $a_{i+1}-a_{i}<\delta / 2$, and construct $\tau$ by induction on $n$ as follows.

For $n=1$, cover $J(p, S)=\{p\}$ with a set type $I^{+}(x) \cap U_{x}$ with $x \in K \cap T_{a_{0}}^{a_{1}}$ and consider the corresponding function $j_{x}$. For a suitable constant $c>0$, the product $c j_{x}$ satisfies both, (ii), (iii) and (iv). To obtain smoothability preserving (i), consider the open covering $\left\{I^{-}\left(S_{a+\delta}\right), I^{+}\left(S_{a+\delta / 2}\right)\right\}$ of $M$, and the first function $0 \leq \mu \leq 1$ of the associated partition of unity (Supp $\mu \subset I^{-}\left(S_{a+\delta}\right)$ ). The required function is just $\tau=c \mu j_{x}$.

Now, assume by induction that the result follows for any chain $a_{0}<\cdots<a_{n-1}$. So, for any $k \leq n-1$, consider $J\left(p, S_{a_{k}}\right)$ and choose a compact set $\hat{K} \subset \operatorname{Int} K$ with $J(p, S) \subset \operatorname{Int} \hat{K}$. Then, there exists a function $\hat{\tau}$ which satisfies condition (i) above for $V=\operatorname{Int} \hat{K} \cap I^{-}\left(S_{a_{k+1}}\right)$ and conditions (ii), (iii), (iv) for $S=S_{a_{k}}$. Now, cover $\hat{K} \cap T_{a_{k}}^{a_{k+1}}$ with a finite number of sets type $I^{+}\left(x^{i}\right) \cap U_{x^{i}}$ with $x^{i} \in K \cap T_{a_{k-1}}^{a_{k+1}}$, and consider the corresponding functions $j_{x^{i}}$.

For a suitable constant $c>0$, the sum $\hat{\tau}+c \sum_{i} j_{x^{i}}$ satisfies (iii) for $S=S_{a_{k+1}}$. This is obvious in $J^{-}\left(S_{a_{k}}\right)$ (for any $c>0$ ), because of the convexity of timelike cones and the reversed triangle inequality. To realize that this can also be obtained in $T_{a_{k}}^{a_{k+1}}$, where $\nabla \tau$ may be non-timelike, notice that the support of $\left.\nabla \hat{\tau}\right|_{T_{a_{k}}^{a_{k+1}}}$ is compact, and it is included in the interior of the support of $\sum_{i} j_{x^{i}}$, where the gradient of the sum is timelike; so, use Lemma 4.1. As $J\left(p, S_{a_{k+1}}\right)$ is compact, conditions (ii), (iv) can be trivially obtained by choosing, if necessary, a bigger $c$.

Finally, smoothability (and (i)), can be obtained again by using the open covering $\left\{I^{-}\left(S_{a_{k+1}+\delta}\right), I^{+}\left(S_{a_{k+1}+\delta / 2}\right)\right\}$ of $M$, and the corresponding first function $\mu$ of the associated partition of unity, i.e., $\tau=\mu\left(\hat{\tau}+c \sum_{i} j_{x^{i}}\right)$.

In order to extend locally defined time functions to a global time function, one cannot use a partition of unity (as stressed in the previous proof, because $\nabla \tau$ is not always timelike when $\mu$ is non-constant). Instead, local time functions must be added directly and, then, coverings as the following will be useful.

Definition 4.3. Let $S$ be a Cauchy hypersurface. A fat cone covering of $S$ is a sequence of pairs of points $p_{i}^{\prime} \ll p_{i}, i \in \mathbb{N}$ such that both $\mathcal{C}^{\prime}=\left\{I^{+}\left(p_{i}^{\prime}\right): i \in \mathbb{N}\right\}$ and $\mathcal{C}=\left\{I^{+}\left(p_{i}\right): i \in \mathbb{N}\right\}$ yield a locally finite covering of $S$.

Proposition 4.4. Any Cauchy hypersurface $S$ admits a fat cone covering $p_{i}^{\prime} \ll$ $p_{i}, i \in \mathbb{N}$.

Moreover, both $\mathcal{C}$ and $\mathcal{C}^{\prime}$ also yield a finite subcovering of $J^{+}(S)$.

Proof. Let $\left\{K_{j}\right\}_{j}$ be a sequence of compact subsets of $S$ satisfying $K_{j} \subset$ Int $K_{j+1}$, $S=\cup_{j} K_{j}$. Each $K_{j} \backslash$ Int $K_{j-1}$ can be covered by a finite number of sets type

\footnotetext{
${ }^{2}$ Along the proof, we will use this lemma only for Cauchy hypersurfaces which are slices of a prescribed time function. However, any Cauchy hypersurface can be written as such a slice for some Cauchy time function. In fact, it is easy to obtain a proof by taking into account that both $I^{+}(S)$ and $I^{-}(S)$ are globally hyperbolic and, thus, admit a Cauchy time function. For details, including the non-trivial case that $S$ is smooth spacelike and $t$ is also required to be temporal, see [5].

${ }^{3}$ Strictly, we will need only the local finiteness of $\mathcal{C}^{\prime}$.
} 
$I^{+}\left(p_{j k}\right), k=1 \ldots k_{j}$ such that $I^{+}\left(p_{j k}\right) \cap S \subset K_{j+1} \backslash K_{j-2}$. Moreover, by continuity of the set-valued function $I^{+}$, this last inclusion is fulfilled if each $p_{j k}$ is replaced by some close $p_{j k}^{\prime} \ll p_{j k}$, and the required pairs $p_{i}^{\prime}\left(=p_{j k}^{\prime}\right), p_{i}\left(=p_{j k}\right)$, are obtained.

For the last assertion, take $q \in J^{+}(S)$ and any compact neighborhood $W \ni q$. As $J^{-}(W) \cap S$ is compact, it is intersected only by finitely many elements of $\mathcal{C}, \mathcal{C}^{\prime}$, and the result follows.

\subsection{Construction of the b-decomposition.}

Definition 4.5. Let $p^{\prime}, p \in T_{a-1}^{a}, p^{\prime} \ll p$. A steep forward cone function (SFC) for $\left(a, p^{\prime}, p\right)$ is a smooth function $h_{a, p^{\prime}, p}^{+}: M \rightarrow[0, \infty)$ which satisfies the following:

(1) $\operatorname{Supp}\left(h_{a, p^{\prime}, p}^{+}\right) \subset J\left(p^{\prime}, S_{a+2}\right)$.

(2) $h_{a, p^{\prime}, p}^{+}>1$ on $S_{a+1} \cap J^{+}(p)$

(3) If $x \in J^{-}\left(S_{a+1}\right)$ and $h_{a, p^{\prime}, p}^{+}(x) \neq 0$, then $\nabla h_{a, p^{\prime}, p}^{+}(x)$ is timelike and pastdirected, and

(4) $g\left(\nabla h_{a, p^{\prime}, p}^{+}, \nabla h_{a, p^{\prime}, p}^{+}\right)<-1$ on $J\left(p, S_{a+1}\right)$.

Now, Proposition 4.2 applied to $S=S_{a+1}, V=I^{-}\left(S_{a+2}\right) \cap I^{+}\left(p^{\prime}\right)$ directly yields:

Proposition 4.6. For all $\left(a, p^{\prime}, p\right)$ there exists an SFC.

The existence of a fat cone covering (Proposition 4.4) allows us to find a function $h_{+}^{a}$ which in some sense globalizes the properties of an SFC.

Lemma 4.7. Choose $a \in \mathbb{R}$ and take any fat cone covering $\left\{p_{i}^{\prime} \ll p_{i} \mid i \in \mathbb{N}\right\}$ for $S=S_{a}$. For every positive sequence $\left\{c_{i} \geq 1 \mid i \in \mathbb{N}\right\}$, the non-negative function $h_{a}^{+}:=(|a|+1) \sum_{i} c_{i} h_{a, p_{i}^{\prime}, p_{i}}^{+}$satisfies:

(1) $\operatorname{Supp}\left(h_{a}^{+}\right) \subset J\left(S_{a-1}, S_{a+2}\right)$.

(2) $h_{a}^{+}>|a|+1$ or $S_{a+1}$.

(3) If $x \in J^{-}\left(S_{a+1}\right)$ and $h_{a}^{+}(x) \neq 0$, then $\nabla h_{a}^{+}(x)$ is timelike and past-directed, and

(4) $g\left(\nabla h_{a}^{+}, \nabla h_{a}^{+}\right)<-1$ on $J\left(S_{a}, S_{a+1}\right)$.

Proof. Obvious.

The gradient of $h_{a}^{+}$will be spacelike at some subset of $J\left(S_{a+1}, S_{a+2}\right)$. So, in order to carry out the inductive process which proves Theorem 1.2. a strengthening of Lemma 4.7 will be needed.

Lemma 4.8. Let $h_{a}^{+} \geq 0$ as in Lemma 4.7. Then there exists a function $h_{a+1}^{+}$ which satisfies all the properties corresponding to Lemma 4.7 and additionally:

$$
g\left(\nabla\left(h_{a}^{+}+h_{a+1}^{+}\right), \nabla\left(h_{a}^{+}+h_{a+1}^{+}\right)\right)<-1 \text { on } J\left(S_{a+1}, S_{a+2}\right)
$$

(so, this inequality holds automatically on all $J\left(S_{a}, S_{a+2}\right)$ ).

Proof. Take a fat cone covering $\left\{p_{i}^{\prime} \ll p_{i} \mid i \in \mathbb{N}\right\}$ for $S=S_{a+1}$. Now, for each $p_{i}$ consider a constant $c_{i} \geq 1$ such that $c_{i} h_{a+1, p_{i}^{\prime}, p_{i}}^{+}+h_{a}^{+}$satisfies inequality (4.1) on $J\left(p_{i}, S_{a+2}\right)$ (see Lemma 4.1). The required function is then $h_{a+1}^{+}=(|a|+$ 2) $\sum_{i} c_{i} h_{a+1, p_{i}^{\prime}, p_{i}}^{+}$

\footnotetext{
${ }^{4}$ This condition is imposed in order to ensure that the finally obtained temporal function is Cauchy. It could be dropped if one looks only for a temporal function and, then, uses Proposition $2.1(2)$.
} 
Now, we have the elements to complete our main proof.

Proof of Theorem 1.2, Consider the function $h_{a}^{+}$provided by Lemma 4.7 for $a=0$, and apply inductively Lemma 4.8 for $a=n \in \mathbb{N}$. Then, we obtain a function $\mathcal{T}^{+}=$ $\sum_{n=0}^{\infty} h_{n}^{+} \geq 0$ with nowhere spacelike gradient, which is a steep temporal function on $J^{+}\left(S_{0}\right)$ with support in $J^{+}\left(S_{-1}\right)$. Analogously, one can obtain a function $\mathcal{T}^{-} \geq 0$ which is a steep temporal function with the reversed time orientation on $J^{-}\left(S_{0}\right)$. So, $\mathcal{T}=\mathcal{T}^{+}-\mathcal{T}^{-}$is clearly a steep temporal function on all $M$.

Moreover, the levels hypersurfaces of $\mathcal{T}$ are Cauchy. In fact, consider any futuredirected causal curve $\gamma$, and reparametrize it with the Cauchy time function $t$. Then,

$\lim _{t \rightarrow \infty} \mathcal{T}(\gamma(t))\left(=\lim _{n \in \mathbb{N}} \mathcal{T}^{+}(\gamma(n+1)) \geq \lim _{n \in \mathbb{N}} h_{n}^{+}(\gamma(n+1))\right)=\infty, \quad \lim _{t \rightarrow \infty} \mathcal{T}(\gamma(t))=-\infty$,

and $\gamma$ crosses all the levels of $\mathcal{T}$, as required.

\section{Appendix}

Clarke [7] developed the following method in order to embed isometrically any manifold $M$ endowed with a semi-Riemannian (or even degenerate) metric $g$ in some semi-Euclidean space $\mathbb{R}_{s}^{N}$. First, he proved that, for some $p \geq 0$, there exists a function $f: M \rightarrow \mathbb{R}_{p}^{p}$ such that the (possibly degenerate) pull-back metric $g(f)$ on $M$ induced from $f$ satisfies $g_{R}=g-g(f)>0$. So, the results for positive definite metrics are applicable to $\left(M, g_{R}\right)$, and one can construct a Riemannian isometric embedding $f_{R}: M \rightarrow \mathbb{R}^{N_{0}}$ ( $f_{R}$ can be constructed from Nash's result, even though Clarke develops a technique to reduce the Nash value for $\left.N_{0}\right)$. Then, the required embedding $i: M \rightarrow \mathbb{R}_{p}^{N}$ is obtained as a product $i(x)=\left(f(x), f_{R}(x)\right)$ for $N=p+N_{0}$.

In Lorentzian signature, Clarke's optimal value for $p$ is 2 . Nevertheless, he claims that, if $(M, g)$ is a globally hyperbolic spacetime, then one can take $p=1$ [7, Lemma 8]. Our purpose in this Appendix is to analyze this question and show:

(A) the required condition $g-g(f)>0$ on $f$ is essentially equivalent to be a steep temporal function, and

(B) the success of the construction of $f$ in [7, Lemma 8] depends on a problem of smoothability, which may have interest in its own right.

In order to make these points clear, we will particularize the proof of 7 , Lemma $8]$ to a very simple case, and will follow most of the notation there. In a previous remark, Clarke assumed that the existence of a temporal function $\tau$ had already been proved, as this question (one of the prominent folk problems of smoothability) seemed true then. In any case, we can now assume that even $\tau$ is Cauchy temporal. Then, consider a globally hyperbolic spacetime which can be written as

$$
\left(\mathbb{R}^{2}, g\right) \quad g=-V^{2} d \tau^{2}+M^{2} d y^{2},
$$

where $(\tau, y)$ are the natural coordinates of $\mathbb{R}^{2}$ and $V, M$ are two positive functions on $\mathbb{R}^{2}$. Easily, a function $f: \mathbb{R}^{2} \rightarrow \mathbb{R}_{1}^{1}\left(=\mathbb{L}^{1}\right)$ satisfies $g-g(f)>0$ if and only if

$$
-V^{2}\left(\partial_{y} f\right)^{2}+M^{2}\left(\partial_{\tau} f\right)^{2}>V^{2} M^{2}
$$

and this is trivially equivalent to $g(\nabla f, \nabla f)<-1$. This proves (A) in our particular example and, taking into account Remark 3.6(1), it seems general. 
Now, consider any smooth function $\sigma \geq 0$ on $\mathbb{R}^{2}$ invariant through the flow of $\nabla \tau$ such that $\sigma^{-1}([0, s])$ is compact for all $s$, and let $Y=\sigma^{-1}([0,1])$; in our simplified example, we can put $\sigma(\tau, y)=|y|^{2}$. Outside $Y$ the two lightlike vector fields,

$$
A_{ \pm}=M \partial_{\tau} \pm V \partial_{\sigma}
$$

are well defined, and equation (5.1) can also be rewritten as

$$
\left(A_{+} f\right)\left(A_{-} f\right)>V^{2} M^{2} \text {. }
$$

So, the crux is to construct a function $f$ which satisfies (5.2) outside $Y$, among other conditions. Clarke's proposal is the following. Let

$$
H^{ \pm}(t, s)=J^{ \pm}\left(\tau^{-1}(0)\right) \cap J^{\mp}\left(\tau^{-1}(t) \cap \sigma^{-1}([0, s])\right) .
$$

After choosing a certain volume element $\omega$, the function $f$ is defined as

$$
f(x)=\int_{H^{+}(\tau(x), \sigma(x))} \omega
$$

whenever $\tau(x)>\epsilon>0$ and outside a neighborhood of $Y$. Notice that $A_{ \pm}$are future directed, and $A_{+}$points outward the region $\sigma^{-1}([0, \sigma(x)])$ at each $x \in M \backslash Y$. So, if $f$ is $C^{1}$, then one would expect $A_{+}(f)>A_{-}(f)>0$. Moreover, Clarke claims that (5.2) can also be achieved by choosing $\omega$ large enough (and eventually, a redefinition of $\tau$ ).

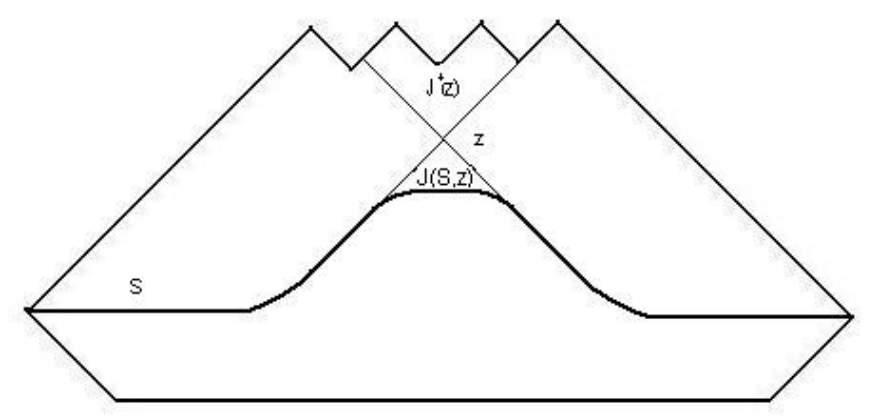

Figure 1. The depicted open subset of $\mathbb{L}^{2}$ is globally hyperbolic, and $S$ a smooth Cauchy hypersurface. Functions $J^{+}(x)$ and $J(S, x)$ are not smooth at $z \in I^{+}(S)$.

To what extent can one assume that $f$ is $C^{1}$ (or, at least, that it can be smoothed to a function which satisfies the required conditions)? For each measurable subset $Z$ of the spacetime manifold, consider its $\omega$-measure $\mu(Z)=\int_{Z} \omega$. In any causally continuous spacetime $M$ it is known that the functions $x \mapsto \mu\left(J^{ \pm}(x)\right)$ are continuous if $\mu(M)<\infty$. Moreover, if $M$ is globally hyperbolic and $S$ is any

\footnotetext{
${ }^{5}$ For $\tau(x)<-\epsilon<0$, the function $f$ is negative and defined dually in terms of $H^{-}$, for $\tau(x)=0$, $f$ is 0 , and a more technical definition is given for $f$ on a neighborhood of $Y \cup \tau^{-1}(0)$. However, this is not relevant for our discussion.
} 
topological Cauchy hypersurface, then $I^{+}(S)$ is a globally hyperbolic spacetime in its own right, and the function $x \mapsto \mu(J(S, x)), x \in I^{+}(S)$, becomes continuous, even if we drop the assumption about the finiteness of $\mu$. Nevertheless, neither the functions $\mu\left(J^{ \pm}(x)\right)$ nor $\mu(J(S, x))$ are smooth in general (see Figure 11). In Clarke's case, the fact that $S=\tau^{-1}(0)$ is not only smooth but spacelike may help to establish smoothness. However, recall that the definition of $f$ also uses the function $\sigma$. Such a $\sigma$ can be defined by taking some auxiliary complete Riemannian metric on $S$, and smoothing along the cut locus the squared distance function to a fixed point $y_{0} \in S$. The behavior of $f$ at the points $x \in M$ such that the boundary of $S \cap J^{\mp}\left(\tau^{-1}(x) \cap \sigma^{-1}([0, \sigma(x)])\right)$ intersects the cut locus may complicate the situation.

Summing up, even assuming (as a necessary element of Clarke's proof) the existence of a temporal function, which was proved in [4, 17] and is proved again in a shorter form for the globally hyperbolic case here, the smoothability of $f$ remains as a non-trivial problem. The solution of this question not only would complete Clarke's proof but also may have interest in its own right.

\section{ACKNOWLEDGEMENTS}

The comments by Professor L. Andersson, who emphasized reference [7, and the careful reading by the referee, are deeply appreciated.

The second author was partially supported by Grants P09-FQM-4496 (J. Andalucía) and MTM2010-18099 (MICINN), both with FEDER funds. The first author was partially funded by CoNaCyT projects 49093 and 82471.

\section{REFERENCES}

[1] C. Bär, N. Ginoux, F. Pfäffle, Wave equations on Lorentzian manifolds and quantization, ESI Lectures in Mathematics and Physics, Eur. Math. Soc. Publ. House, Zürich, 2007. MR2298021 (2008j:58041)

[2] J.K. Beem, P.E. Ehrlich, K.L. Easley, Global Lorentzian geometry, Monographs Textbooks Pure Appl. Math. 202, Dekker Inc., New York, 1996. MR.1384756 (97f:53100)

[3] A.N. Bernal and M. Sánchez, On smooth Cauchy hypersurfaces and Geroch's splitting theorem, Commun. Math. Phys. 243 (2003) 461-470. MR2029362 (2004j:53086)

[4] A.N. Bernal and M. Sánchez, Smoothness of time functions and the metric splitting of globally hyperbolic spacetimes, Commun. Math. Phys. 257 (2005) 43-50. MR2163568(2006g:53105)

[5] A.N. Bernal and M. Sánchez, Further results on the smoothability of Cauchy hypersurfaces and Cauchy time functions, Lett. Math. Phys. 77 (2006) 183-197. MR2254187(2007k:53109)

[6] A.N. Bernal and M. Sánchez, Globally hyperbolic spacetimes can be defined as "causal" instead of "strongly causal", Class. Quant. Grav. 24 (2007) 745-750. MR 2294243 (2007m:53089)

[7] C.J.S. Clarke, On the global isometric embedding of pseudo-Riemannian manifolds, Proc. Roy. Soc. London Ser. A 314 (1970) 417-428. MR0259813 (41:4445)

[8] R. Geroch, Domain of dependence, J. Math. Phys. 11 (1970) 437-449. MR0270697(42:5585)

[9] R. E. Greene, Isometric embeddings of Riemannian and pseudo-Riemannian manifolds, Memoirs of the American Mathematical Society, No. 97 American Mathematical Society, Providence, R.I. 1970 iii+63 pp. MR0262980 (41:7585)

[10] Q. Han and J-X. Hong, Isometric embedding of Riemannian manifolds in Euclidean spaces, Mathematical Surveys and Monographs, 130, American Mathematical Society, Providence, RI, 2006. MR2261749 (2008e:53055)

[11] N.H. Kuiper, On $C^{1}$-isometric imbeddings. I, II, Nederl. Akad. Wetensch. Proc. Ser. A. 58 (Indag. Math. 17) (1955) 545-556. MR0075640 (17:782c)

[12] E. Minguzzi and M. Sánchez, The causal hierarchy of spacetimes, in Recent developments in pseudo-Riemannian geometry, ESI Lect. Math. Phys., Eur. Math. Soc. Publ. House, Zürich, 2008, p. 299-358. MR2436235 (2010b:53128) 
[13] O. Müller, The Cauchy problem of Lorentzian minimal surfaces in globally hyperbolic manifolds, Ann. Global Anal. Geom. 32 (2007), no. 1, 67-85. MR.2334944(2009b:53099)

[14] J. Nash, The imbedding problem for Riemannian manifolds, Ann. of Math. (2) 63 (1956), 20-63. MR0075639 (17:782b)

[15] B. O'Neill, Semi-Riemannian Geometry with applications to Relativity, Academic Press Inc., 1983. MR719023 (85f:53002)

[16] G. Ruzzi, Punctured Haag duality in locally covariant quantum field theories. Comm. Math. Phys. 256 (2005) 621-634. MR2161274 (2006m:81165)

[17] M. Sánchez, Causal hierarchy of spacetimes, temporal functions and smoothness of Geroch's splitting. A revision, Contemporanea Matematica 28 (2005) 127-155. MR 2196783 (2006m:53111)

[18] J. T. Schwartz, On Nash's implicit functional theorem, Comm. Pure Appl. Math. 13 (1960) 509-530. MR0114144 (22:4971)

[19] H. Stephani, D. Kramer, M. MacCallum, C. Hoenselaers, E. Herlt, Exact solutions of Einstein's field equations, Cambridge Monographs on Mathematical Physics (2003). MR 2003646 (2004h:83017)

Instituto de Matemáticas, Universidad Nacional Autónoma de México, Campus MoreLia, C. P. 58190 Morelia, MichoacÁn, MÉXico

E-mail address: olaf@matmor.unam.mx

Current address: Fakultät für Mathematik, Universität Regensburg, Universitätsstrasse 31, D-93059 Regensburg, Germany

E-mail address: Olaf.Mueller@mathematik.uni-regensburg.de

Departamento de Geometría y Topología, Facultad de Ciencias, Universidad de Granada, Campus Fuentenueva s/n, 18071 Granada, Spain

E-mail address: sanchezm@ugr.es 